\title{
Establishment and evaluation of a simulated high-altitude hypoxic brain injury model in SD rats
}

\author{
YA HOU $^{1 *}$, XIAOBO WANG $^{1 *}$, XIAORUI CHEN ${ }^{1}$, JING ZHANG $^{2}$, XIAOPENG AI $^{1}$, YUSHENG LIANG $^{1}$, \\ YANGYANG YU ${ }^{2}$, YI ZHANG ${ }^{2}$, XIANLI MENG ${ }^{1,3}$, TINGTING KUANG ${ }^{2}$ And YAO HU ${ }^{1,4}$ \\ ${ }^{1}$ Department of Pharmacology of Chinese Materia Medica, School of Pharmacy; ${ }^{2}$ Department of Tibetan Medicine, \\ Ethnic Medicine College; ${ }^{3}$ Institute of Traditional Chinese Medicine Innovation; ${ }^{4}$ Department of Sports Medicine, \\ Institute of Physical Education, Chengdu University of Traditional Chinese Medicine, \\ Wenjiang, Chengdu, Sichuan 611137, P.R. China
}

Received May 29, 2018; Accepted January 25, 2019

DOI: $10.3892 / \mathrm{mmr} .2019 .9939$

\begin{abstract}
This study was conducted to establish a stable hypobaric hypoxia brain injury model. SD rats were randomly separated into control and model groups, and placed outside or inside of a hypobaric chamber, respectively. Subsequent to $24 \mathrm{~h}$ anoxic exposure, plasma superoxide dismutase (SOD), malondialdehyde (MDA), glutathione (GSH), oxidized glutathione (GSSG) and lactate dehydrogenase (LDH) were measured using commercial biochemical kits. Hematoxylin-eosin (H\&E), Nissl's and terminal deoxynucleotidyl transferase dUTP nick end labeling (TUNEL) staining were used to observe the morphology of neurons in the hippocampus. The protein expression levels of apoptotic protease activating factor-1 (Apaf-1), hypoxia inducible factor-1 $\alpha$ (HIF-1 $\alpha$ ), caspase-3, cleaved caspase-3, Bcl-2-associated X protein (Bax) and cytochrome c (cyto-c) were detected using western blot and immunohistochemistry analyses. Hypoxic substantially induced morphological lesions in the hippocampus concomitant with the physical behavioral performance deficit. Furthermore, hypoxia markedly exacerbated the levels of MDA, LDH and GSSG, and restrained GSH $(\mathrm{P}<0.01)$ and SOD $(\mathrm{P}<0.05)$ levels compared with the control group. In addition,
\end{abstract}

Correspondence to: Dr Tingting Kuang, Department of Tibetan Medicine, Ethnic Medicine College, Chengdu University of Traditional Chinese Medicine, 1166 Liutai Street, Wenjiang, Chengdu, Sichuan 611137, P.R. China

E-mail: 13424821@qq.com

Dr Yao Hu, Department of Sports Medicine, Institute of Physical Education, Chengdu University of Traditional Chinese Medicine, 1166 Liutai Street, Wenjiang, Chengdu, Sichuan 611137, P.R. China E-mail: 392402056@qq.com

*Contributed equally

Key words: high-altitude hypoxia, hypobaric chamber, brain injury, Sprague-Dawley rats, hypoxia-inducible factor- $1 \alpha$, mitochondrial apoptosis hypoxia significantly induced the protein expression of Apaf-1, HIF-1 $\alpha$, caspase- 3 , cleaved caspase-3, Bax and Cyto-c $(\mathrm{P}<0.01)$ compared with the control group. Finally, a lower number and volume of Nissl bodies were verified in the hypoxic group. TUNEL results demonstrated a greater number of apoptotic cells in the hypoxic group. The present study demonstrates a model of rat hypoxic brain injuries induced by a hypobaric chamber at 9,000 $\mathrm{m}$ for $24 \mathrm{~h}$. Furthermore, the redox enzyme, HIF-1 $\alpha$ and mitochondrial apoptosis-associated protein, along with H\&E and Nissl's staining, may be applied to evaluate the degree of injury.

\section{Introduction}

Inhabitants who live at $>3,000$ m elevation may experience damage to the heart, brain, lungs and other organs due to the low inspired $\mathrm{PO}_{2}$ and the reduced barometric pressure (1-4). Furthermore, Zhang et al (5) reported that 4,500 m elevation is the turning point for high-altitude polycythemia (HAPC) prevalence in Tibetan communities. However, the Mount Everest $(8,848 \mathrm{~m})$, being well renowned as the roof of the world, appears to be close to the limit of human tolerance to hypoxia $(6,7)$. Hence, these previous data provide evidence to suggest that the three altitudes of 3,000, 4,500 and 8,848 $(\sim 9,000)$ m are points of elevation which are notable in research conducted on plateau hypoxia.

In previous years, an increasing number of lowlanders have travelled to high-altitude areas for recreation or work all year round. Unfortunately, individuals travelling to high-altitude areas from low-altitude areas have the potential risk of developing mountain sickness due to exposure to a hypobaric hypoxia environment at a high altitude, including acute mountain sickness, high-altitude pulmonary edema, high-altitude cerebral edema, chronic mountain sickness, high-altitude pulmonary hypertension and HAPC (8-11). These diseases can be life-threatening. Therefore, the improvement of the endurance of humans in resisting hypoxia and reducing hypoxia-induced organ damage is a global challenge.

Considering the issues mentioned above, the purpose of the present study was to establish an equably simulated acute plateau anoxia brain injury model of Sprague-Dawley (SD) 
rats and reliable methodology validation. The present study aimed to provide a foundation for the investigation into the mechanisms and molecular-targeted therapeutic drugs used for hypoxic brain injury.

\section{Materials and methods}

Reagents. Urethane was purchased from Aladdin Shanghai Biochemical Technology Co., Ltd (Shanghai, China). Hematoxylin and eosin (H\&E) were provided by Thermo Fisher Scientific, Inc. (Waltham, MA, USA). The total extraction sample kit (cat. no. AR0101-30), BCA protein assay kit (cat. no. AR0146), sodium dodecyl sulfate-polyacrylamide gel electrophoresis (SDS-PAGE) protein sample buffer $2 \mathrm{X}$ (denaturation; cat. no. AR0131), broad spectrum protease inhibitor (cat. no. AR1182-1), broad spectrum phosphatase inhibitor (cat. no. AR1183), terminal deoxynucleotidyl transferase dUTP nick end labeling (TUNEL) apoptosis detection kit I-POD (cat. no. MK1025) and primary antibodies against Bcl-2-associated X protein (Bax; cat. no. BA0315), apoptotic protease activating factor-1 (Apaf-1; cat. no. BA2373), hypoxia inducible factor (HIF)-1 $\alpha$ (cat. no. PB0245) and cytochrome $c$ (cyto-c; cat. no. A03529) were obtained from Wuhan Boster Biological Technology, Ltd. (Wuhan, China). Caspase-3 (cat. no. \#9662), and cleaved caspase-3 (cat. no. \#9661) antibodies were procured from Cell Signaling Technology, Inc. (Danvers, MA, USA). Antibodies against $\beta$-actin (cat.no. GB11001) and horseradish peroxidase-conjugated goat anti-rabbit immunoglobulin G (H+L; cat. no. GB23303) were obtained from Servicebio (Wuhan, China). Ultrasignal electrochemiluminescence (ECL) substrate (cat. no. 4AW011-100) was obtained from 4A Biotech Co., Ltd (Beijing, China). Enzyme-linked immunosorbent assay (ELISA) kits for lactate dehydrogenase (LDH; cat. no. A020-2), superoxide dismutase (SOD; cat. no. A001-3), malondialdehyde (MDA; cat. no. A003-1) and glutathione/oxidized glutathione (GSH/GSSG; cat. no. A061-1) were provided by Nanjing Jiancheng Bioengineering Institute (Nanjing, China).

Animals. A total of 12 male specific pathogen-free SD rats (weighing 200-220 g, 7 weeks old) were obtained from Chengdu Dashuo Experimental Animal Co., Ltd (Chengdu, China) and were maintained in a $12 \mathrm{~h}$ light/dark cycle at room temperature $\left(23 \pm 2^{\circ} \mathrm{C}\right)$ in $50-60 \%$ relative humidity. They were randomly divided into two groups of six rats each and were fed chow and water ad libitum. Animals were maintained for one week for the purpose of acclimatization prior to the experimental treatment. All rats were anesthetized using an intraperitoneal injection of $20 \%$ urethane $(0.5 \mathrm{ml} / 100 \mathrm{~g})$.

Ethical statement. All procedures were performed in accordance with the Animal Care Guidelines, conforming to the Health Guide for the Care and Use of Laboratory Animals (12), and the protocol of this investigation was ethically approved by the Animal Research Ethics Committee of Chengdu University of Traditional Chinese Medicine (Chengdu, China).

Simulated high-altitude hypoxia brain injury model. Based on a previous investigation on HAPC or high-altitude cerebral injury rat models (13) and other similar studies (14-17), a modified version of an animal model of acute hypobaric hypoxia brain injury model was developed. In brief, subsequent to acclimatization for $30 \mathrm{~min}$ in an animal decompression chamber (AVIC Guizhou Fenglei Aviation Armament Co., Ltd., Guizhou, China; cat. no. FLYDWC50-II C), which is able to simulate a $\sim 10,000 \mathrm{~m}$ global atmospheric condition by the reduction of ambient barometric pressure in combination with precise monitoring temperature $\left(-40-60^{\circ} \mathrm{C}\right)$ and relative humidity (0-100\%). An anoxic environment was simulated by evacuating the air from the chamber using powerful vacuum pumps. Fresh air was allowed to circulate, and the air flow was maintained inside the chamber at 0.91 per min to replenish consumed $\mathrm{O}_{2}$ and remove the produced $\mathrm{CO}_{2}$. The hypoxic rats were subjected to the following protocols: 3,000 and 4,500 m altitude at a speed of $5 \mathrm{~m} / \mathrm{s}$ for $30 \mathrm{~min}$ and subsequent simulated 9,000 m altitude for $23 \mathrm{~h}$. The chamber was maintained at a temperature of $15-17^{\circ} \mathrm{C}$ and a relative humidity of $55-60 \%$. Following hypobaric exposure for $24 \mathrm{~h}$, the elevation was immediately lowered at a speed of $5 \mathrm{~m} / \mathrm{s}$. Meanwhile, the control group was subject to similar conditions at an altitude of $\sim 600$ m outside of the chamber, in order to simulate a pure high altitude environment that is lacking in oxygen and at a low pressure rather compared with a low altitude environment with normal air circulation. The protocol of the present study design is presented in Fig. 1.

Determination of oxidative stress indicators in serum. The levels of SOD, MDA, GSH, GSSG and LDH in the sera of six rats that had undergone $24 \mathrm{~h}$ simulated high-altitude hypoxic treatment were measured using ELISA kits according to the manufacturers' protocol. The absorbance values of SOD and LDH were read at $450 \mathrm{~nm}$, MDA at $532 \mathrm{~nm}$ and GSSG and total (T-)GSH at $405 \mathrm{~nm}$ using a full-wavelength scanning multifunction reading meter (Varioskan Flash; Thermo Fisher Scientific, Inc., Waltham, MA, USA). The concentration of GSH was calculated by the formula: GSH=T-GSH-2xGSSG. The values were expressed in terms of units SOD per ml, nmol MDA per ml, $\mu \mathrm{mol} \mathrm{GSH}$ and GSSG per 1 and units LDH per 1 .

Hematoxylin \& eosin $(H \& E)$ staining and immunohistochemical detection. The serum was acquired from the abdominal aorta of the rats. Subsequently, the whole brain was removed from the skull and fixed using $100 \mathrm{ml} \mathrm{4 \%}$ paraformaldehyde at a $7.2 \mathrm{pH}$ at $25^{\circ} \mathrm{C}$ for $24 \mathrm{~h}$. The paraffin-embedded sections of the hippocampal tissue were sliced into $5 \mu \mathrm{m}$ thick sections using cryotome (cat. no. RM2235; Leica Microsystems GmbH, Wetzlar, Germany), baked at $60^{\circ} \mathrm{C}$ overnight and dewaxed using xylene I and II for $20 \mathrm{~min}$. Then, the sections were stained with hematoxylin for $30 \mathrm{~min}$ at $25^{\circ} \mathrm{C}$, washed with water for $20 \mathrm{~min}$, stained with eosin for $5 \mathrm{~min}$ at $25^{\circ} \mathrm{C}$, dehydrated with $100,95,80$ and $70 \%$ ethanol for $5 \mathrm{~min}$ each, cleared using xylene and mounted with neutral balsam.

For immunohistochemical assessment, the protocol was performed as described previously (18). In brief, subsequent to deparaffinization, blocking with $10 \%$ goat serum at $25^{\circ} \mathrm{C}$ for $15 \mathrm{~min}$, the $4-\mu \mathrm{m}$-thick sections were incubated with primary antibodies (1:400 rabbit anti-HIF-1 $\alpha$ and 1:1,000 anti-Caspase-3) in $1 \%$ bovine serum albumin, $0.3 \%$ triton $\mathrm{X}-100$ and $0.1 \%$ sodium azide in phosphate buffered saline (PBS) at $4^{\circ} \mathrm{C}$ overnight, washed three times for 3 min each with 


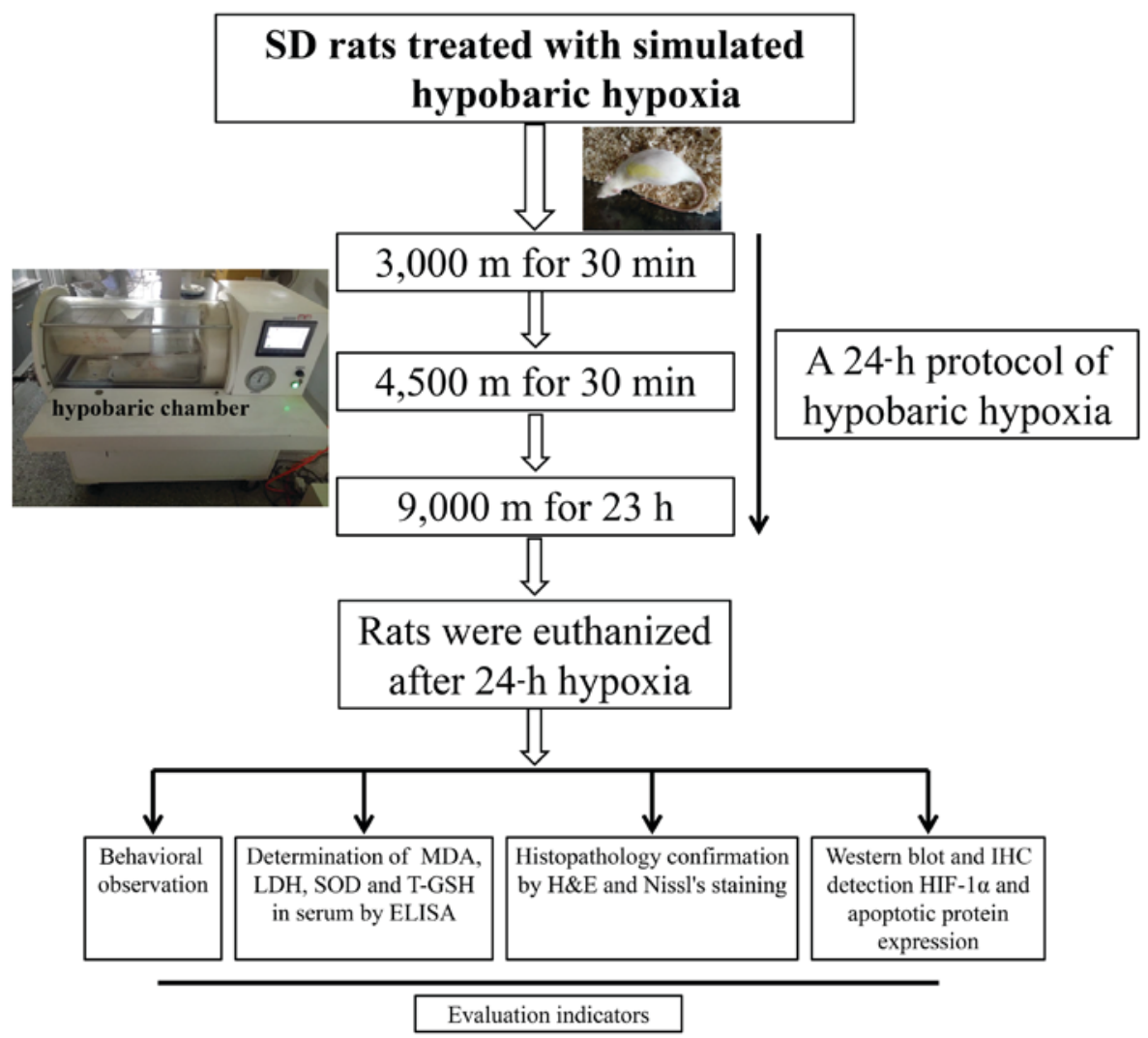

Figure 1. Study design of the experimental procedure. SD, Sprague-Dawley; MDA, malondialdehyde; LDH, lactate dehydrogenase; SOD, superoxide dismutase; T-GSH, total glutathione; ELISA, enzyme-linked immunosorbent assay; H\&E, hematoxylin and eosin; IHC, immunohistochemistry; HIF-1 $\alpha$, hypoxia inducible factor- $1 \alpha$.

PBS, and subsequently incubated with goat anti-rabbit antibody conjugated to horseradish peroxidase (cat. no. 18149A01, OriGene Technologies, Inc., Beijing, China) diluted 1:300 in bull serum albumin blocking buffer for $2 \mathrm{~h}$ at room temperature, followed by use of a 3,3'-diaminobenzidine (DAB) kit according to the manufacturers' protocol.

Finally, images were acquired using a Leica microscopic imaging system (cat. no. DM1000; Leica Microsystems GmbH, Wetzlar, Germany) using a CX22 light microscope with x200 magnification for positive stains (Olympus Corporation, Tokyo Japan) to record the lesions in the CA1, CA2, CA3 and dentate gyrus (DG) regions of the hippocampus. The levels of HIF- $1 \alpha$ and caspase- 3 in the CA1 region were scored using the Image-Pro Plus software version 6.0 (Media Cybernetics, Inc., Rockville, MD, USA). The experiment was repeated three times.

Western blot analysis. Frozen brains were homogenized in cold whole cell lysis buffer (cat. no. AR0101-30; Wuhan Boster Biological Technology, Ltd., Wuhan, China) and centrifuged at $12,000 \mathrm{x} \mathrm{g}$ for $10 \mathrm{~min}$ at $4^{\circ} \mathrm{C}$. The total protein concentration was determined using a BCA protein assay kit according to the manufacturer's protocol. Subsequent to mixing with the loading buffer and boiling at $100^{\circ} \mathrm{C}$ for $5 \mathrm{~min}, 40 \mu \mathrm{g}$ protein per lane were separated using 10\% SDS-PAGE.

Proteins were electrophoretically transferred onto polyvinylidene difluoride membranes and then incubated with the corresponding primary antibodies overnight at $4^{\circ} \mathrm{C}$ subsequent to blocking using 5\% skim milk for $1 \mathrm{~h}$ at room temperature.
The primary antibodies included Apaf-1 (1:300), HIF-1 $\alpha$ $(1: 1,000), \beta$-actin $(1: 1,000)$, caspase- 3 and cleaved caspase- 3 $(1: 1,000), \operatorname{Bax}(1: 300)$ and cyto-c $(1: 200)$ overnight at $4^{\circ} \mathrm{C}$. Membranes were washed with PBS with $0.1 \%$ Tween-20 and subsequently incubated with horseradish peroxidase-conjugated goat anti-rabbit immunoglobulin $\mathrm{G}$ secondary antibodies (1:1,000; cat. no. GB23303; Wuhan Goodbio Technology Co., Ltd., Wuhan, China) for $2 \mathrm{~h}$ at room temperature. The blots were developed using ultrasignal Western ECL substrate (4A Biotech Co., Ltd.) and visualized using ChampChemi 610 Plus (Beijing Sage Creation Science Co., Ltd., Beijing, China). The relative expression levels of the protein bands of interest were quantified and normalized to $\beta$-actin using Image-Pro Plus software version 6.0 (Media Cybernetics, Inc.).

Nissl and TUNEL staining. To detect the histomorphological changes in the hippocampal neurons, Nissl staining was performed as previously described (19). In brief, the $4-\mu \mathrm{m}$ sections were incubated with $0.1 \%$ toluidine blue for $10 \mathrm{~min}$ at room temperature, rinsed in double distilled water followed by treatment with 70 and $95 \%$ ethanol, dehydrated in $100 \%$ ethanol, cleared using xylene and coated with neutral resin.

Apoptosis was evaluated using a TUNEL apoptosis detection kit according to the manufacturers' protocol. In brief, the $5 \mu \mathrm{m}$ paraffin-embedded brain sections were fully dewaxed and hydrated, soaked in $3 \%$ hydrogen peroxide for $10 \mathrm{~min}$ at $25^{\circ} \mathrm{C}$, and washed with distilled water three times for 2 min each time. Subsequently, the sections were treated with proteinase $\mathrm{K}$ solution at $37^{\circ} \mathrm{C}$ for $15 \mathrm{~min}$ and washed 
with $0.01 \mathrm{M}$ tris buffered saline (TBS) thrice for 2 min each time. Then the sections were tagged for $2 \mathrm{~h}$ at $37^{\circ} \mathrm{C}$ with $20 \mu \mathrm{l}$ mixed labeling buffer ( $1 \mu 1$ TdT:1 $\mu 1$ DIG-dUTP:18 $\mu$ l labeling buffer) for each section and washed with $0.01 \mathrm{M}$ TBS thrice for 2 min each time. Subsequently, they were blocked at room temperature for $30 \mathrm{~min}$ with $50 \mu \mathrm{l}$ blocking reagent. A total of $50 \mu \mathrm{l}$ biotinylated anti-digoxin antibody (1:100) was added to the sections at $37^{\circ} \mathrm{C}$ for $30 \mathrm{~min}$ and washed with $0.01 \mathrm{M}$ TBS thrice for $2 \mathrm{~min}$ each time. Then, the sections were incubated with $50 \mu \mathrm{l}$ streptavidin biotin complexes $(1: 100)$ at $37^{\circ} \mathrm{C}$ for $30 \mathrm{~min}$, washed with $0.01 \mathrm{M}$ TBS four times for $5 \mathrm{~min}$ each time, treated using $\mathrm{DAB}$ at $20^{\circ} \mathrm{C}$ for $10 \mathrm{~min}$, subjected to mild hematoxylin restaining at $25^{\circ} \mathrm{C}$ for $2 \mathrm{~min}$, rinsed in $0.01 \mathrm{M} \mathrm{TBS}$ followed with distilled water, dehydrated in $100 \%$ ethanol, cleared using xylene and coated using neutral resins. Blocking reagent, biotinylated anti-digoxin antibody, streptavidin biotin complexes were all taken from apoptosis detection kit I-POD (cat. no. MK1025; Wuhan Boster Biological Technology, Ltd.) and were used strictly according with the manufacturer's protocols. All sections were randomly observed under a CX22 light microscope with a x200 magnification (Olympus Corporation). The number of Nissl's bodies and the TUNEL positive apoptotic cell rate were calculated using Image-Pro Plus software version 6.0 (Media Cybernetics, Inc.).

Statistical analysis. Statistical analysis was conducted using SPSS 17.0 (SPSS, Inc., Chicago, IL, USA). Results were presented as the mean \pm standard deviation ( $n=6$ with replicas for each sample). The statistical analysis was performed using analysis of variance followed by a Tukey's post hoc test. $\mathrm{P}<0.05$ was considered to indicate a statistically significant difference.

\section{Results}

Physical behavioral performance. Following $30 \mathrm{~min}$ acclimatization in a multifunctional hypobaric chamber, a hypoxic protocol was conducted on six rats, while the control group of rats were simultaneously treated outside of the chamber as a parallel control group. During the first $5 \mathrm{~min}$, the abnormal activities or behavioral performance of the rats were observed in the hypoxic group. Briefly, an increased activity frequency, increased respiratory rate, head-up and neck extension, hind legs straight and nasal flaring allegro were observed in the hypoxic group compared with the control group. In addition, the physical activities reduced gradually as the hypoxic time extended. Following $0.5 \mathrm{~h}$, all the rats stood still, huddled together, trembled and fell to one side occasionally. Ultimately, the semiclosed eyes and extremely fast belly breathing continued until the end of the hypoxic treatment. However, no abnormal activities or behavioral performance were observed in the control group. No rats succumbed to mortality either among the control or hypoxic group in a 24-h hypoxic duration at 9,000 $\mathrm{m}$. Hence, the present hypoxic protocol that was applied is able to induce abnormal activities or affect the behavioral performance of rats.

Effect of hypoxia on oxidative stress indicators in serum. The antioxidant activities of MDA, GSSG, LDH and reduced SOD along with GSH in the rats stimulated by hypobaric hypoxia are summarized in Table I. The results revealed significantly
Table I. MDA, GSH, GSSG, SOD and LDH activities in the serum of rats (mean \pm standard deviation, $n=6$ ).

\begin{tabular}{lcr}
\hline Parameter & Control group & Model group \\
\hline SOD $(\mathrm{U} / \mathrm{ml})$ & $25.38 \pm 0.69$ & $21.65 \pm 0.14^{\mathrm{a}}$ \\
$\mathrm{MDA}(\mathrm{nmol} / \mathrm{ml})$ & $12.91 \pm 0.40$ & $26.12 \pm 1.62^{\mathrm{b}}$ \\
$\mathrm{GSH}(\mu \mathrm{mol} / \mathrm{l})$ & $10.89 \pm 0.30$ & $1.74 \pm 0.40^{\mathrm{b}}$ \\
$\mathrm{GSSG}(\mu \mathrm{mol} / \mathrm{l})$ & $1.79 \pm 0.43$ & $6.21 \pm 0.67^{\mathrm{b}}$ \\
$\mathrm{LDH}(\mathrm{U} / \mathrm{l})$ & $572.77 \pm 30.66$ & $872.50 \pm 37.21^{\mathrm{b}}$ \\
\hline
\end{tabular}

MDA, malondialdehyde; LDH, lactate dehydrogenase; SOD, superoxide dismutase; GSH, glutathione; GSSG, oxidized glutathione. ${ }^{\mathrm{a}} \mathrm{P}<0.05$ and ${ }^{\mathrm{b}} \mathrm{P}<0.01$ vs. control group.

higher levels of serum MDA, LDH and GSSG $(\mathrm{P}<0.01)$, and significantly lower levels of serum SOD $(\mathrm{P}<0.05)$ and GSH $(\mathrm{P}<0.01)$ in the model group compared with that in the control group. The statistical results are presented in Fig. 2.

Hypobaric hypoxia induces neuronal damage in the hippocampus of rats. H\&E staining revealed that the nerve cell arrangement was well organized in the hippocampus CA1, CA2, CA3 and DG regions in the control group. Conversely, a remarkable neuron loss, cellular swelling, widened pericellular spaces and shrunken neurons with darkly stained pyknotic nuclei in the hippocampi and cortex following hypoxic treatment were observed in the hypoxic group (Fig. 3). As presented in Fig. $4 \mathrm{~A}$ and $\mathrm{B}$, the number of surviving neurons stained by Nissl's presented a significantly decreased integrated optical density (IOD) in the CA1 region in hypobaric hypoxic rats compared with that in the control group $(\mathrm{P}<0.01)$. In addition, the TUNEL positive apoptotic cell rate was significantly higher in the hypoxic group compared with that in the control group $(\mathrm{P}<0.01)$.

Hypoxia enhances the expression of Apaf-1, HIF-1 $\alpha$, caspase-3, cleaved caspase-3, Bax and cyto-c. The HIF-1 $\alpha$ and apoptosis-associated protein expression levels are presented in Figs. 4 and 5. Western blot analysis revealed that the protein expression levels of Apaf-1, HIF-1 $\alpha$, caspase-3, cleaved caspase-3, Bax and cyto-c in the model group were significantly higher compared with that in the control group $(\mathrm{P}<0.01$; Fig. 4C and D). Significantly stronger immunopositive caspase-3 and HIF-1 $\alpha$ levels were also observed in the model group compared with that in the control group $(\mathrm{P}<0.01$; Fig. 5).

\section{Discussion}

Behavioral investigation in animals has revealed that abnormal activity performances are associated with brain damage (20-22). A previous study has revealed a series of affected physical activities in rats subjected to middle cerebral artery occlusion and reperfusion injury (23). In the present experiment, hypoxia-associated abnormal performances, including nasal flaring allegro, head-up and neck extension, hind legs straightening and an increased respiratory rate were 

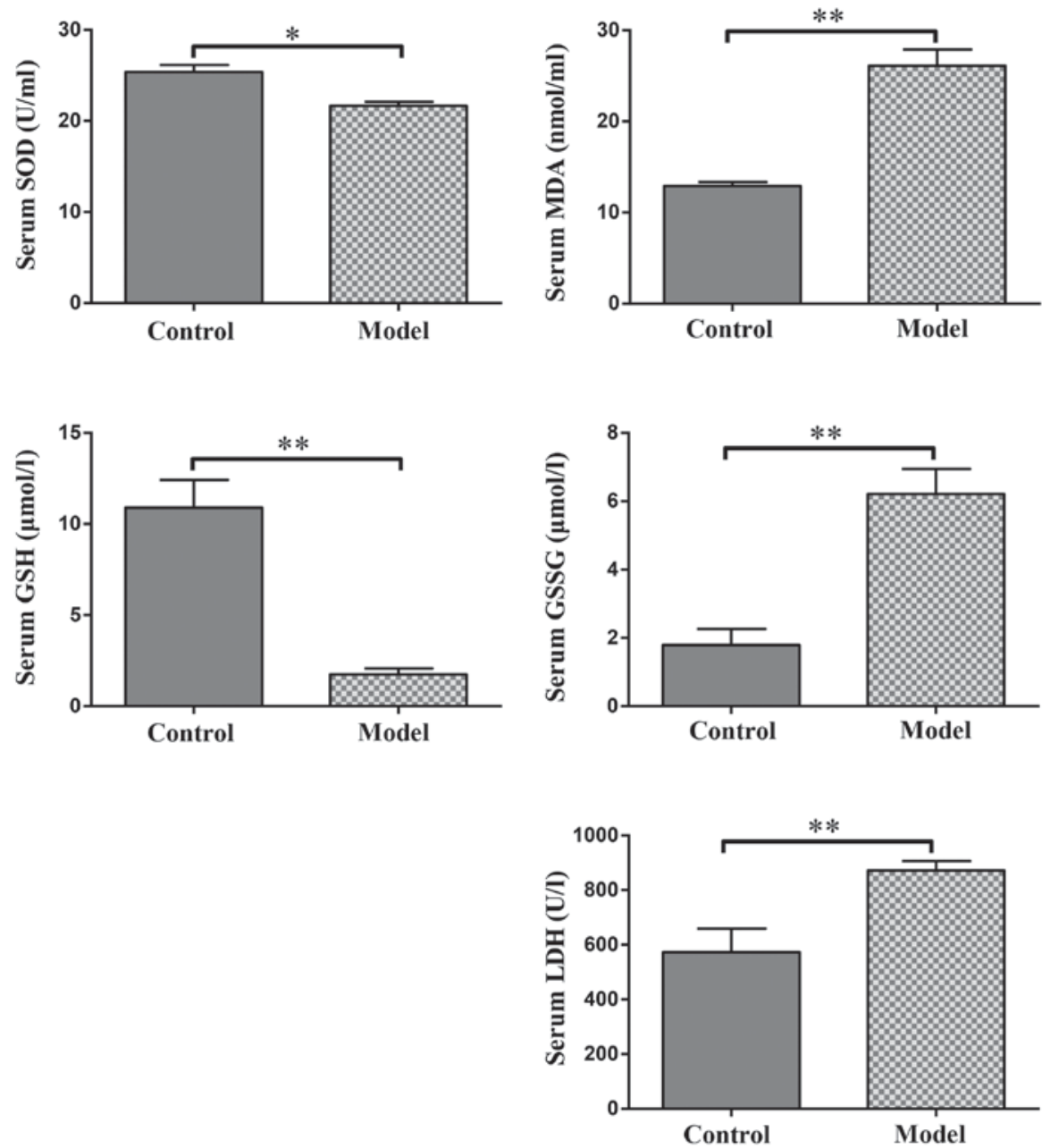

Figure 2. Effect of hypoxia on serum SOD, MDA, GSH, GSSG and LDH levels. The values are presented as the mean \pm standard deviation. "P $<0.05$ and ${ }^{* *} \mathrm{P}<0.01$ with comparisons shown by lines. MDA, malondialdehyde; LDH, lactate dehydrogenase; SOD, superoxide dismutase; GSH, glutathione; GSSG, oxidized glutathione.
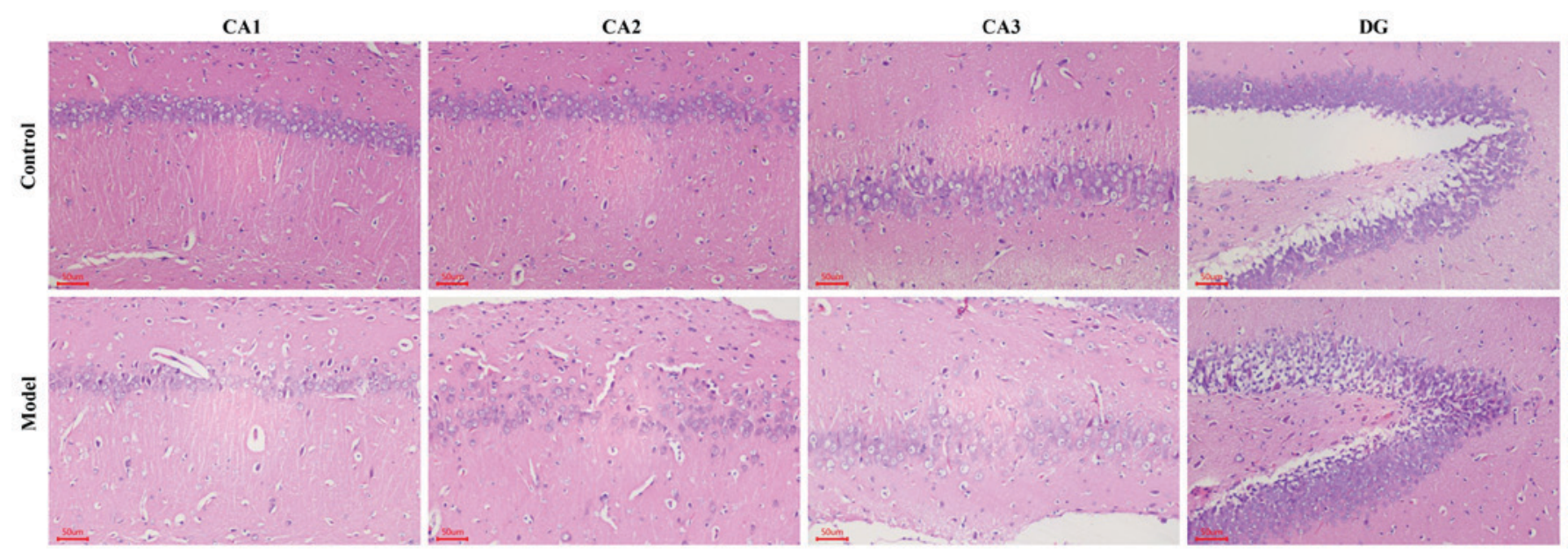

Figure 3. Effect of hypoxia demonstrated using hematoxylin and eosin staining in the hippocampus area. Neuron cells in the CA1, CA2, CA3 and DG regions of the hippocampus. Scale bar, $50 \mu \mathrm{m}$. Original magnification, x200. DG, dentate gyrus.

also presented in hypobaric-hypoxia-induced rat brain injuries. During the hypoxic period, biomarkers in the serum or injured tissues including increased MDA, GSSG and LDH levels and decreased SOD and GSH levels have been demonstrated to alter substantially (24-27). The results of the present study indicate an elevation of MDA, GSSG and LDH levels in the serum compared with that in the control group, whereas SOD and GSH levels decreased in the model. The results are 

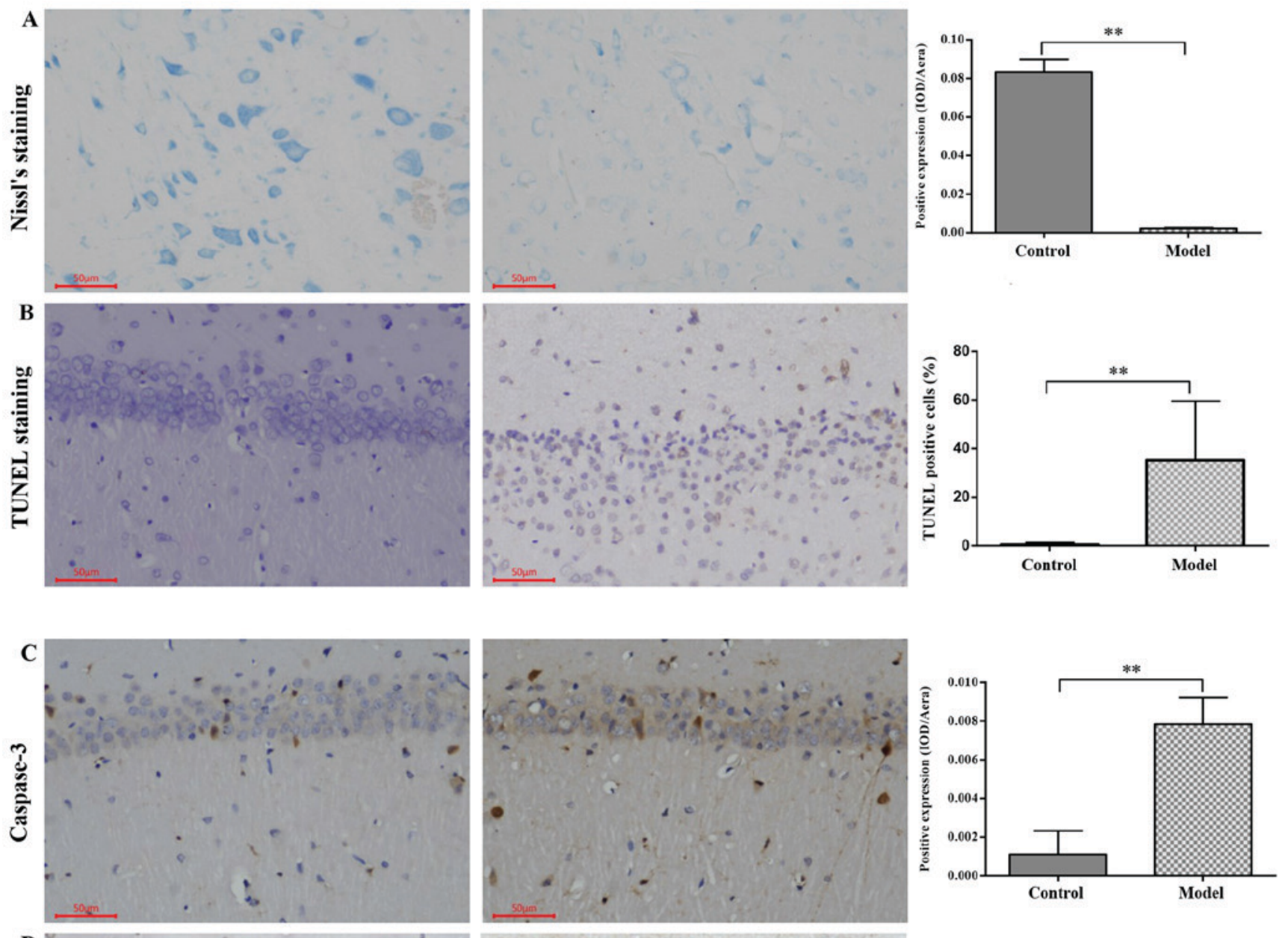

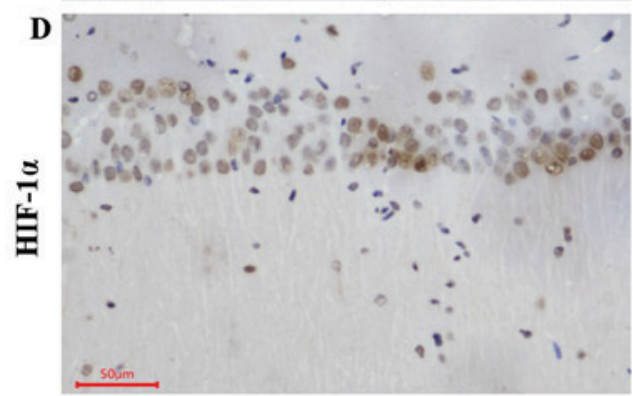

Control

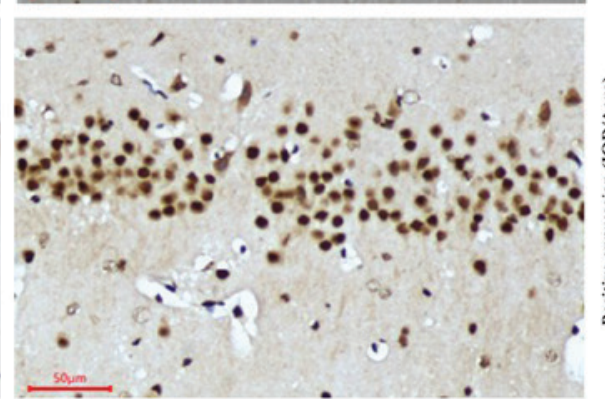

Model

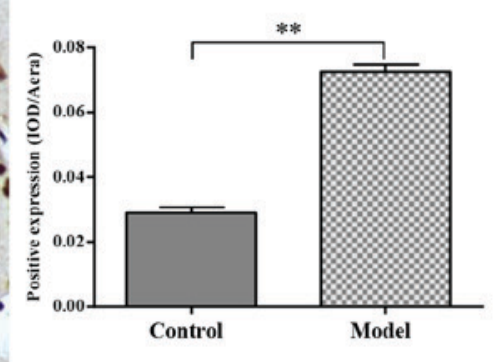

Figure 4. Immunohistochemistry for caspase-3 and HIF-1 $\alpha$ protein expression and Nissl's and TUNEL staining in the CA1 region in the hippocampus following hypoxia. (A) Nissl-stained neurons in the CA1 region of the hippocampus of SD rats. (B) TUNEL-positive apoptotic cells in the CA1 region of the hippocampus of SD rats. (C) Caspase-3 expression examined using immunohistochemical analysis and quantified. (D) HIF-1 $\alpha$ expression examined using immunohistochemical analysis and quantified. Scale bar, $50 \mu \mathrm{m}$. Original magnification, $\mathrm{x} 200$. The values are presented as the mean \pm standard deviation. ${ }^{* *} \mathrm{P}<0.01$ with comparisons shown by lines. HIF-1 $\alpha$, hypoxia inducible factor-1 $\alpha$; TUNEL, terminal deoxynucleotidyl transferase dUTP nick end labeling; SD, Sprague-Dawley; IOD, integrated optical density.

presented in Table I and Fig. 2. Therefore, this indicates that the buffering system or redox enzyme in serum may be used for diagnosis of early brain injury.

Mounting evidence suggests that hypoxic or ischemic insult may induce morphological deterioration in the rat hippocampus, including sparse cell arrangement in the pyramidal layer of the hippocampus and karyopyknosis at the microscale (28-30). The present results of H\&E staining (Fig. 3) of the rat hippocampus revealed similar pathological phenomena in the CA1, CA2, CA3 and DG in the hypoxic rat hippocampus. In addition, substantially decreased quantities and sizes of Nissl bodies in the hippocampus were observed in the hypoxic group, which were consistent with the results of previous studies (Fig. 4C) (31-33).

HIFs, which consist of HIF-1 and HIF-2 isoforms, respond to the prevailing level of oxygen. Functioning as a master switch in the human response to hypoxia, a substantially higher protein expression of HIF-1 $\alpha$ is produced during hypoxia. Subsequently, it translocates from the cytoplasm to the nucleus (34-35). Finally, the HIF-1 $\alpha$ protein binds to specific DNA sequences of downstream targets, thereby controlling the transcription rate of $>200$ genes (36-39). Zheng et al (40) reported that salidroside induced the inhibition of HIF-1 $\alpha$ protein degradation rather than its gene overexpression. As a result, HIF-1 $\alpha$ protein 


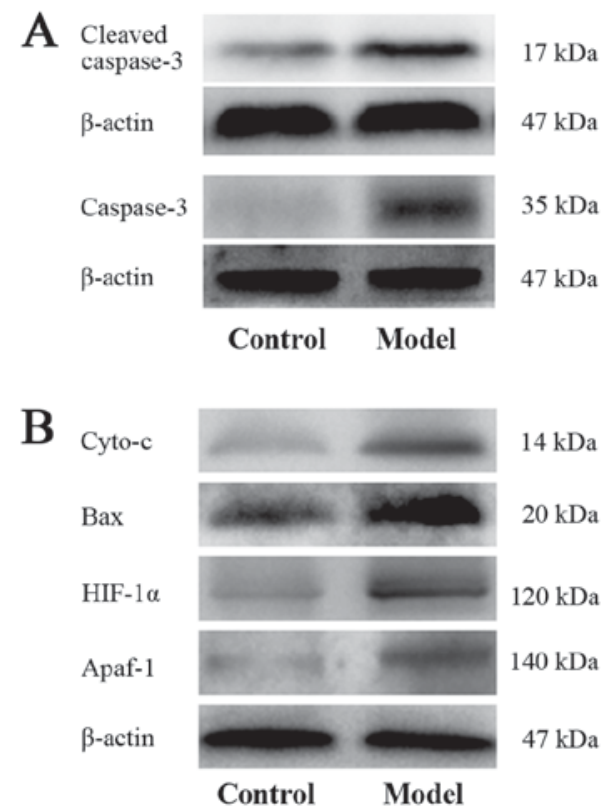

C

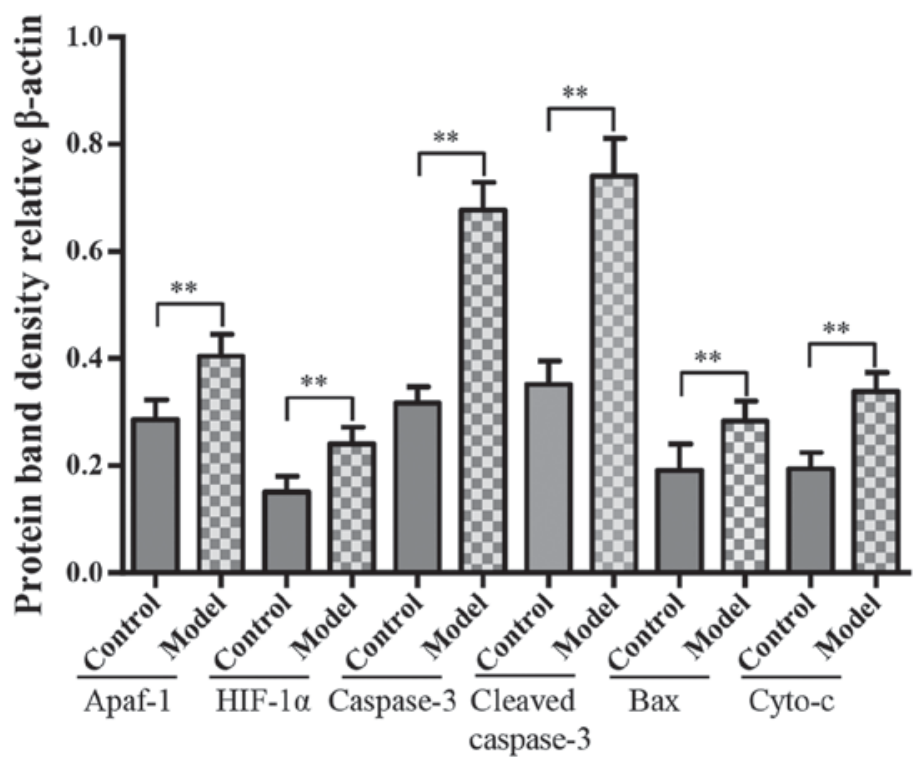

Figure 5. Hypoxia-activated mitochondrial apoptosis pathway. Western blot analysis for (A) caspase-3 and cleaved caspase-3, (B) Apaf-1, HIF-1 $\alpha$, Bax and cyto-c protein level in each group, $\mathrm{n}=6$. (C) Quantified western blot analysis results. The values are presented as mean \pm standard deviation. ${ }^{* * *} \mathrm{P}<0.01$ with comparisons shown by lines. Apaf-1, apoptotic protease activating factor-1; HIF-1 $\alpha$, hypoxia inducible factor-1 $\alpha$; Bax, Bcl-2-associated X protein; cyto-c, cytochrome c.

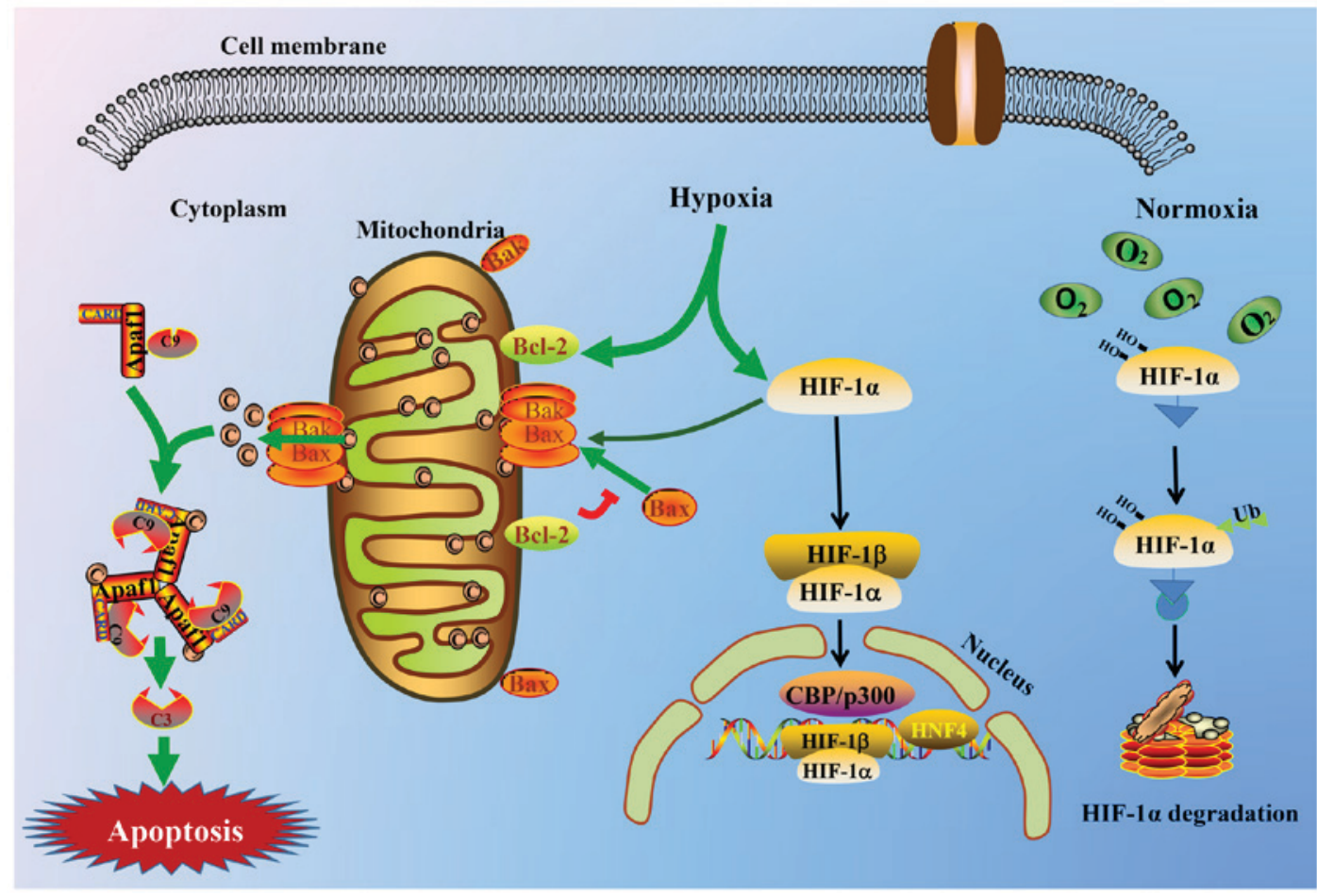

Figure 6. Schematic view of HIF-1 $\alpha$ and the intrinsic triggering pathway of the apoptotic signaling cascade. Hypoxia stimulates the HIF-1 $\alpha$ and mitochondrial apoptotic signaling pathway and triggers the release of cyto-c from the mitochondrial matrix to the cytoplasm, thereby binding to the Apaf-1 protein, inducing its conformational change and activating it. Thus, the apoptosome consisting of cyto-c/Apaf-1/caspase-9 is yielded and is able to cleave or activate the executioner caspase-3. The activated caspase-3 complex then results in apoptosis. C9, caspase-9; C3, caspase-3; HIF-1 $1 \alpha$, hypoxia inducible factor-1 $\alpha$; cyto-c, cytochrome c; Apaf-1, apoptotic protease activating factor-1; CARD, caspase recruitment domain containing; Bak, Bcl2 antagonist/killer; Bax, Bcl-2-associated X protein; Bcl-2, Bcl2, apoptosis regulator; CBP, CREB binding protein; HIF-1 $\beta$, hypoxia inducible factor-1 $\beta$; HNF4, hepatocyte nuclear factor $4 \alpha$.

expression tended to increase. However, a higher gene expression of HIF-1 $\alpha$ stimulated by hypoxia in addition to salidroside was confirmed previously $(41,42)$. Thus, HIF-1 $\alpha$ may be viewed as a notable molecule that may be affected by drugs or oxygen concentration, while the affected biosynthesis or degradation of the HIF-1 $\alpha$ protein in vivo requires further investigation. In 
the present study, the increased accumulation of the HIF-1 $\alpha$ protein was detected by western blot analysis in the hypoxic group compared with that in the control group. Furthermore, it was previously reported that hypoxia or ischemic brain injuries induce a decrease of mitochondrial membrane potential (43). Increased protein levels of cyto-c, Bax and Apaf-1 were also demonstrated in the present study. Thus, the apoptotic body consisting of Apaf-1, cyto-c and caspase- 9 in the cytoplasm accelerates the process of cell apoptosis through activating the caspase-dependent apoptotic signaling pathway (44). A graphic illustration of the involved molecular mechanism is presented in Fig. 6. The protein expression levels of HIF-1 $\alpha$, caspase-3 and cleaved caspase-3 were assessed using immunohistochemistry and western blot analysis in the present study. The results are presented in Figs. 4C-D and 5. Cyto-c, Bax and Apaf-1 protein expression levels were consistently increased in the hypoxic group compared with that in the control group; the results are presented in Fig. 5B. Furthermore, TUNEL staining was used to evaluate whether hypobaric hypoxia induced neuronal apoptosis, as presented in Fig. 4B, a large number of positive staining apoptotic cells appeared in hippocampal neurons of the hypoxic group.

In conclusion, a hypobaric hypoxia brain injury rat model induced by a multifunctional hypobaric chamber was established successfully at 9,000 $\mathrm{m}$ for $24 \mathrm{~h}$. The redox enzymes in the serum, HIF-1 $\alpha$ and mitochondrial apoptosis-associated proteins, along with $\mathrm{H} \& \mathrm{E}$ and Nissl's staining, may be applied to evaluate the degree of a hypoxic injury. This simulated high-altitude hypoxia brain injury model in SD rats would be beneficial for progressing high-altitude hypoxia drug discovery. In addition, the increased HIF-1 $\alpha$ protein expression induced by high-altitude hypoxia may stimulate the expression of the microRNA-210 gene, thereby inhibiting the expression of iron-sulfur cluster assembly enzyme $1 / 2$ and COX10, Heme A:Farnesyltransferase cytochrome $c$ oxidase assembly factor, which affect the process of mitochondrial energy metabolism $(45,46)$. Furthermore, the hypoxic-activated phosphoinositide-3-kinase/protein kinase B signaling pathway may also increase HIF-1 $\alpha$ overexpression, and bind to the hypoxia response element in the nucleus and trigger downstream target gene expression, including vascular endothelial growth factor, erythropoietin, heme oxygenase 1, inducible nitric oxide synthase and nuclear factor- $\kappa \mathrm{B}(47)$. Thus, it is reasonable to believe that HIF- $1 \alpha$ is a core component of the body's response to hypoxia. The degree and duration of hypoxia determine its protein synthesis and degradation. The association between HIF- $1 \alpha$ and hypoxia, and additional treatment strategies urgently require further investigation and development.

\section{Acknowledgements}

Not applicable.

\section{Funding}

The present study was supported by the National Natural Science Foundation of China (grant no. 81203000), National Natural Science Foundation of China (grant no. 81403187), State Administration of Traditional Chinese Medicine of the People's Republic of China, Special Scientific Research in the Traditional Chinese Medicine field (grant no. 201507002) and Supported by Sichuan Science and Technology Program (grant no. 19YYJC1922).

\section{Availability of data and materials}

The datasets used and/or analyzed during the current study are available from the corresponding author on reasonable request.

\section{Authors' contributions}

YH designed the present study, performed the animal experiments, analyzed the data and wrote the manuscript. XW, XC and $\mathrm{JZ}$ performed the animal experiments, the physiological test and the H\&E staining. XA, TK and YH analyzed the data, organized the images and improved the language. YL and YY performed the protein detection and immunohistochemical analysis. YZ and XM performed serum biochemical indexes detection, Nissl's and TUNEL staining. All authors read and approved the final manuscript.

\section{Ethics approval and consent to participate}

The protocol of this investigation was ethically approved by the Animal Research Ethics Committee of Chengdu University of Traditional Chinese Medicine (Chengdu, China).

\section{Patient consent for publication}

Not applicable.

\section{Competing interests}

The authors declare that they have no competing interests.

\section{References}

1. Young AJ, Berryman CE, Kenefick RW, Derosier AN, Margolis LM, Wilson MA, Carrigan CT, Murphy NE, Carbone JW, Rood JC, et al: Altitude acclimatization alleviates the hypoxia-induced suppression of exogenous glucose oxidation during steady-state aerobic exercise. Front Physiol 9: 830, 2018.

2. Gonzalez Garay A, Molano Franco D, Nieto Estrada VH, Martí-Carvajal AJ and Arevalo-Rodriguez I: Interventions for preventing high altitude illness: Part 2. Less commonly-used drugs. Cochrane Database Syst Rev 3: CD012983, 2018.

3. Paul S, Gangwar A, Bhargava K, Khurana P and Ahmad Y: Diagnosis and prophylaxis for high-altitude acclimatization: Adherence to molecular rationale to evade high-altitude illnesses. Life Sci 203: 171-176, 2018.

4. Xiang K, Ouzhuluobu, Peng Y, Yang Z, Zhang X, Cui C, Zhang H, Li M, Zhang Y, Bianba, et al: Identification of a Tibetan-specific mutation in the hypoxic gene EGLN1 and its contribution to high-altitude adaptation. Mol Biol Evol 30: 1889-1898, 2013.

5. Zhang H, He Y, Cui C, Ouzhuluobu, Baimakangzhuo, Duojizhuoma, Dejiquzong, Bianba, Gonggalanzi, Pan Y, et al: Cross-altitude analysis suggests a turning point at the elevation of 4,500 $\mathrm{m}$ for polycythemia prevalence in Tibetans. Am J Hematol 92: E552-E554, 2017.

6. Gonggalanzi, Labasangzhu, Nafstad P, Stigum H, Wu T, Haldorsen $\varnothing \mathrm{D}$, Ommundsen $\mathrm{K}$ and Bjertness E: Acute mountain sickness among tourists visiting the high-altitude city of Lhasa at 3658 m above sea level: A cross-sectional study. Arch Public Health 74: 23, 2016.

7. West JB: High-altitude medicine. Lancet Respir Med 3: 12-13, 2015.

8. Basnyat B and Murdoch DR: High-altitude illness. Lancet 361: 1967-1974, 2003 
9. Azad P, Stobdan T, Zhou D, Hartley I, Akbari A, Bafna V and Haddad GG: High-altitude adaptation in humans: From genomics to integrative physiology. J Mol Med (Berl) 95: 1269-1282, 2017.

10. Davis C and Hackett P: Advances in the prevention and treatment of high altitude illness. Emerg Med Clin North Am 35: 241-260, 2017.

11. Horscroft JA, Kotwica AO, Laner V, West JA, Hennis PJ, Levett DZH, Howard DJ, Fernandez BO, Burgess SL, Ament Z, et al: Metabolic basis to Sherpa altitude adaptation. Proc Natl Acad Sci USA 114: 6382-6387, 2017.

12. Ward PA, Blanchard RJ and Bolivar V: Recognition and alleviation of distress in laboratory animals. National Academies Press 44: 380, 2007.

13. Zhang Y, Meng X, Wu W, Lai X, Wang Y, Zhang J and Wang Z: Duoxuekang, a traditional Tibetan medicine, reduces hypoxia-induced high-altitude polycythemia in rats. A Sponsored Supplement to Science 338: 63-64, 2012.

14. Zhang XY, Zhang XJ, Xv J, Jia W, Pu XY, Wang HY, Liang H, Zhuoma-Lamao and Lu DX: Crocin attenuates acute hypobaric hypoxia-induced cognitive deficits of rats. Eur J Pharmacol 818: 300-305, 2018

15. Gong G, Yin L, Yuan L, Sui D, Sun Y, Fu H, Chen L and Wang X: Ganglioside GM1 protects against high altitude cerebral edema in rats by suppressing the oxidative stress and inflammatory response via the PI3K/AKT-Nrf2 pathway. Mol Immunol 95 . 91-98, 2018.

16. Song TT, Bi YH, Gao YQ, Huang R, Hao K, Xu G, Tang JW, Ma ZQ, Kong FP, Coote JH, et al: Systemic pro-inflammatory response facilitates the development of cerebral edema during short hypoxia. J Neuroinflammation 13: 63-76, 2016.

17. Guo P, Luo H, Fan Y, Luo Y and Zhou Q: Establishment and evaluation of an experimental animal model of high altitude cerebral edema. Neurosci Lett 547: 82-86, 2013

18. Gu J, Su S, Guo J,Zhu Y,Zhao M and Duan JA: Anti-inflammatory and anti-apoptotic effects of the combination of Ligusticum chuanxiong and Radix Paeoniae against focal cerebral ischaemia via TLR4/MyD88/MAPK/NF- $\mathrm{BB}$ signalling pathway in MCAO rats. J Pharm Pharmacol 70: 268-277, 2018

19. Wang P, Xie ZD, Xie CN, Lin CW, Wang JL, Xuan LN, Zhang CW, Wang Y, Huang ZH and Teng HL: AMP-activated protein kinase-dependent induction of autophagy by erythropoietin protects against spinal cord injury in rats. CNS Neurosei Ther 24: 1185-1195, 2018.

20. Song H, Konan LM, Cui J, Johnson CE, Langenderfer M, Grant D Ndam T, Simonyi A, White T, Demirci U, et al: Ultrastructural brain abnormalities and associated behavioral changes in mice after low-intensity blast exposure. Behav Brain Res 347: 148-157, 2018 .

21. Kaplan GB,Leite-Morris KA, Wang L, Rumbika KK,Heinrichs SC, Zeng X, Wu L, Arena DT and Teng YD: Pathophysiological bases of comorbidity: Traumatic brain injury and post-traumatic stress disorder. J Neurotrauma 35: 210-225, 2018.

22. Mancuso M, Abbruzzese L, Canova S, Landi G, Rossi S and Santarnecchi E: Transcranial random noise stimulation does not improve behavioral and neurophysiological measures in patients with subacute vegetative-unresponsive wakefulness state (VS-UWS). Front Hum Neurosci 11: 524, 2017.

23. Zhao Q, Wang X, Chen A, Cheng X, Zhang G, Sun J, Zhao Y, Huang $\mathrm{Y}$ and $\mathrm{Zhu} \mathrm{Y}$ : Rhein protects against cerebral ischemic-/reperfusion-induced oxidative stress and apoptosis in rats. Int J Mol Med 41: 2802-2812, 2018.

24. Ren H, Meng X, Yin J, Sun J, Huang Q and Yin Z: Ganoderma lucidum polysaccharide peptide attenuates skin flap ischemiareperfusion injury in a thioredoxin-dependent manner. Plast Reconstr Surg 142: 23e-33e, 2018.

25. Zhang X, Rui L, Wang M, Lian H and Cai L: Sinomenine attenuates chronic intermittent hypoxia-induced lung injury by inhibiting inflammation and oxidative stress. Med Sci Monit 24 $1574-1580,2018$

26. Yang G, Min D, Yan J, Yang M and Lin G: Protective role and mechanism of snakegourd peel against myocardial infarction in rats. Phytomedicine 42: 18-24, 2018.

27. Wu H, Luo D, Li C, Zhang H, Shunxian A, Zhang Y and Sun C: Chicoric acid improves heart and blood responses to hypobaric hypoxia in tibetan yaks. Am J Chin Med 46: 339-355, 2018.

28. Yan YT, Li SD, Li C, Xiong YX, Lu XH, Zhou XF, Yang LQ, $\mathrm{Pu} \mathrm{LJ}$ and Luo HY: Panax notoginsenoside saponins Rb1 regulates the expressions of $\mathrm{Akt} / \mathrm{mTOR} / \mathrm{PTEN}$ signals in the hippocampus after focal cerebral ischemia in rats. Behav Brain Res 345: 83-92, 2018
29. Ramli Y, Alwahdy AS, Kurniawan M, Juliandi B, Wuyung PE and Susanto YDB: Intravenous versus intraarterial transplantation of human umbilical cord blood mononuclear cells for brain ischemia in rats. Hayati J Biosci 24: 187-194, 2017.

30. Sun J, Wang F, Li H, Zhang H, Jin J, Chen W, Pang M, Yu J, He Y, Liu J and Liu C: Neuroprotective effect of sodium butyrate against cerebral ischemia/reperfusion injury in mice. Biomed Res Int 2015: 395895, 2015

31. Zuo D, Lin L, Liu Y, Wang C, Xu J, Sun F, Li L, Li Z and Wu Y: Baicalin attenuates ketamine-induced neurotoxicity in the developing rats: Involvement of $\mathrm{PI} 3 \mathrm{~K} / \mathrm{Akt}$ and $\mathrm{CREB} / \mathrm{BDNF} / \mathrm{Bcl}-2$ pathways. Neurotox Res 30: 159-172, 2016.

32. Wu Z, Wu P, Zuo X, Yu N, Qin Y, Xu Q, He S, Cen B, Liao W and Ji A: LncRNA-N1LR enhances neuroprotection against ischemic stroke probably by inhibiting $\mathrm{p} 53$ phosphorylation. Mol Neurobiol 54: 7670-7685, 2017.

33. Zhang JZ, Jing L, Ma Y, Guo FY, Chang Y and Li PA: Monosialotetrahexosy-1 ganglioside attenuates diabetesenhanced brain damage after transient forebrain ischemia and suppresses phosphorylation of ERK $1 / 2$ in the rat brain. Brain Res 1344: 200-208, 2010.

34. Bahrami A, Atkin SL, Majeed M and Sahebkar A: Effects of curcumin on hypoxia-inducible factor as a new therapeutic target. Pharmacol Res 137: 159-169, 2018

35. Gonzalez FJ, Xie C and Jiang C: The role of hypoxia-inducible factors in metabolic diseases. Nat Rev Endocrinol 15: 21-32, 2018.

36. Choudhry $\mathrm{H}$ and Harris AL: Advances in hypoxia-inducible factor biology. Cell Metab 27: 281-298, 2018.

37. West JB: Physiological effects of chronic hypoxia. N Engl J Med 376: 1965-1971, 2017.

38. Lee KE and Simon MC: SnapShot: Hypoxia-inducible factors Cell 163: 1288-1288.e1,2015.

39. West JB: High altitude medicine. Am J Respir Crit Care Med 186 1229-1237, 2012

40. Zheng KY, Zhang ZX, Guo AJ, Bi CW, Zhu KY, Xu SL, Zhan JY, Lau DT, Dong TT, Choi RC and Tsim KW: Salidroside stimulates the accumulation of HIF-1 $\alpha$ protein resulted in the induction of EPO expression: A signaling via blocking the degradation pathway in kidney and liver cells. Eur J Pharmacol 679: 34-39, 2012.

41. Long Z, Li J and Meng X: The protective effects of active ingredients isolated from Rhodiola on the myocardial cell damage induced by hypoxia/glucose deprivation. Pharmacology and Clinics of Chinese Materia Medica 26: 24-25, 2010 (In Chinese).

42. Wang Y, Zhang Y, Feng X, Meng X, Long Y and Zhang J: Bioactive chemical constituents from Rhodiola crenulata and effect on hypoxia inducible factor- $1 \alpha$ expression. West China J Pharmaceutical Sci 24: 021-024, 2009 (In Chinese).

43. Hu Y, Lv X, Zhang J and Meng X: Comparative study on the protective effects of salidroside and hypoxic preconditioning for attenuating anoxia-induced apoptosis in pheochromocytoma (PC12) cells. Med Sci Monit 22: 4082-4091, 2016.

44. Zhao Q, Cheng X, Wang X, Wang J, Zhu Y and Ma X Neuroprotective effect and mechanism of Mu-Xiang-You-Fang on cerebral ischemia-reperfusion injury in rats. J Ethnopharmacol 192: 140-147, 2016.

45. Qiu J, Zhou XY, Zhou XG, Cheng R, Liu HY and Li Y: Neuroprotective effects of microRNA-210 against oxygen-glucose deprivation through inhibition of apoptosis in PC12 cells. Mol Med Rep 7: 1955-1959, 2013.

46. He M, Lu Y, Xu S, Mao L,Zhang L, Duan W, Liu C, Pi H, Zhang Y, Zhong M, et al: MiRNA-210 modulates a nickel-induced cellular energy metabolism shift by repressing the iron-sulfur cluster assembly proteins ISCU1/2 in Neuro-2a cells. Cell Death Dis 5: e1090, 2014.

47. Ye Z, Guo Q, Xia P, Wang N, Wang E and Yuan Y: Sevoflurane postconditioning involves an up-regulation of HIF-1 $\alpha$ and HO-1 expression via PI3K/Akt pathway in a rat model of focal cerebral ischemia. Brain Res 1463: 63-74, 2012.

This work is licensed under a Creative Commons Attribution-NonCommercial-NoDerivatives 4.0 International (CC BY-NC-ND 4.0) License. 